\title{
Liquid rivulet moved by a gas flow in a minichannel
}

\author{
Vladimir V. Kuznetsov ${ }^{1}$ and Yulia O. Kabova ${ }^{2,3, a}$ \\ ${ }^{1}$ Lavrentyev Institute of Hydrodynamics, SB RAS, 630090, 15 Lavrentyev prosp., Novosibirsk, Russia \\ ${ }^{2}$ Institute of Thermophysics, SB RAS, 630090, 1 Lavrentyev prosp., Novosibirsk, Russia \\ ${ }^{3}$ Novosibirsk State University of Architecture and Civil Engineering, 630008, 113 Leningradskaya Str., \\ Novosibirsk, Russia
}

\begin{abstract}
The mathematical model of steady-state flow of incompressible and nonisothermal liquid rivulet moved by a gas in an inclined minichannel was developed. Action of van der Waals and capillary forces, as well as gravity and tangential stress at the gas-liquid interface are taking into account. Numerically it is found out that rivulet formation gives a significant disturbing effect on the gas velocity distribution. It is shown that at the rivulet profile clearly visible three-phase contact line and region with practically constant inclination could be seen near the thin liquid film areas. The angle of this inclination could be considered as a contact angle. Region with practically constant derivative of the function $\mathrm{h}(\mathrm{y})$ with respect to $\mathrm{y}$, near the contact line confirms this statement.
\end{abstract}

\section{Introduction}

Rivulet flows with and without phase-change are abundant in nature and play an important role in our daily life. Fast development in semiconductor technology, including shrinking feature size, increasing transistor density and faster circuit speeds have resulted in greater non-uniformity of on-chip power dissipation, generating localized submillimeter hot spots often exceeding $1 \mathrm{~kW} / \mathrm{cm}^{2}$ in heat flux. Rivulets driven by gravity or forced by gas/vapor flow in narrow channels (100-300 $\mu \mathrm{m})$ are assumed to provide very high heat transfer intensity especially near the contact line area of the fluid on the heated surface $[1,2,3,4,5]$. Rivulet flows represent a promising approach to thermal management of advanced semiconductor devices with high power dissipation, in particularly for space applications: energy production, electronic cooling devices, life support systems and waste water treatment for long duration space exploration missions [6]. Understanding of fundamental aspects of rivulet dynamics might provide a way to control these flows. In [7], a model of a rivulet flow down an inclined substrate was proposed under assumption that the free-surface curvature of the rivulet is constant. In [8] a rivulet flow down an inclined cylinder was considered. The rivulet and gas flow in minichannel were theoretically investigated under conditions of variable gravity in [9]. The mathematical model describes steady-state isothermal rivulets with prescribed contact angle. In general it was found that the flow dynamics at microgravity differs significantly from that at normal gravity. Profile of the rivulet and gas-liquid interface deformations are affected significantly by the flow rates and gravity.

\footnotetext{
${ }^{\text {a }}$ Corresponding author: kabova@itp.nsc.ru
} 


\section{Problem statement}

Let us consider an incompressible and nonisothermal liquid rivulet moved by a gas flow in an inclined minichannel with rectangular cross-section of certain width $l$ and height $d$, Fig. 1 . The liquid and gas motions are assumed to be laminar and steady state and temperature distribution on the bottom wall of the channel is assumed to be permanent along the entire length of the rivulet. The film motion is determined by the shear stress, induced by the gas flow on the free surface, and gravity.
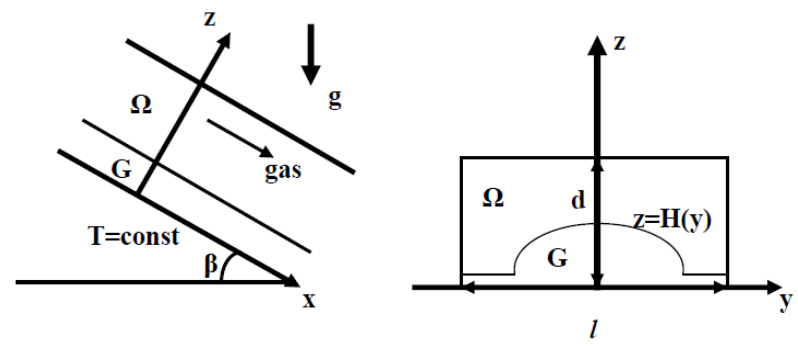

Figure 1. A sketch of the physical model.

Let the liquid and the gas occupy the following areas: $G=\{-\infty<x<\infty,-l / 2<y<l / 2,0<z<H(y)\}$ and $\Omega=\{-\infty<x<\infty,-l / 2<y<l / 2, H(y)<z<d\}$, correspondingly. The flows in the liquid and gas phases are described by the Navier-Stokes equations for incompressible fluid. Thus, in the areas $G$ and $\Omega$ we have the following equations:

$$
\begin{gathered}
\rho(\boldsymbol{v} \cdot \nabla) \boldsymbol{v}=-\nabla p+\rho \boldsymbol{g}+\mu \Delta \boldsymbol{v}, \nabla \cdot \boldsymbol{v}=0 \\
\rho_{g}\left(\boldsymbol{v}_{g} \cdot \nabla\right) \boldsymbol{v}_{g}=-\nabla p_{g}+\rho_{g} \boldsymbol{g}+\mu_{g} \Delta \boldsymbol{v}_{g}, \nabla \cdot \boldsymbol{v}_{g}=0 .
\end{gathered}
$$

Here, $\mathbf{v}$ and $\mathbf{v}_{g}$ are the velocity vectors; $p$ and $p_{g}$ are the pressure distributions in the liquid and the gas $\boldsymbol{g}=(|\boldsymbol{g}| \sin \beta, 0,|\boldsymbol{g}| \cos \beta)$ is the free fall acceleration, $\rho, \rho_{g}$ - densities of the liquid and the gas, $\mu, \mu_{g}$ - dynamic viscosities of the liquid and the gas, $\beta$ - inclination angle of the channel to the horizon. Boundary conditions for system (1)-(2) will take the form:

at $z=0$ and $y= \pm l / 2, z<H( \pm l / 2)$

$$
v=0,
$$

at $z=d$ and $y= \pm l / 2, z>H( \pm l / 2)$

$$
v_{g}=0,
$$

and at $z=H(y)$

$$
\begin{aligned}
& \boldsymbol{v}=\boldsymbol{v}_{g}, \\
& \left(P-P_{g}\right) \boldsymbol{n}=2 \sigma K \boldsymbol{n}-\frac{A}{6 \pi h^{3}} \boldsymbol{n},
\end{aligned}
$$

where $\sigma$ is the surface tension coefficient, $K$ is the mean curvature of the film surface $z=H(y)$, $P_{i j}=-p \delta_{i j}+\mu\left(\partial v_{i} / \partial x_{j}+\partial v_{j} / \partial x_{i}\right), \quad P_{g i j}=-p_{g} \delta_{i j}+\mu_{g}\left(\partial v_{g i} / \partial x_{j}+\partial v_{g j} / \partial x_{i}\right) \quad\left(\delta_{i j}-\right.$ the Kronecker symbols $), \boldsymbol{n}=\left(0,-H^{\prime} / \sqrt{1+H^{\prime 2}}, 1 / \sqrt{1+H^{\prime 2}}\right)$ - normal vector to the surface $z=H(y)$, A - Hamaker constant. Disjoining pressure is taken into account in (6). Modeling of the rivulet form and main features of the flow near the three-phase contact lines should take into account the thin liquid film 
appearing in the vicinity of the contact line, where the action of the van der Waals forces is significant [10]. Let us assume that the bottom wall of the channel is smooth, and thin liquid film covers it all. The height of this film is fast decreasing moving off the rivulet and has no effect on the results obtained.

\section{Development of the mathematical model}

The lubrication-type mathematical model based on the predominant velocity direction approach could be built. Let us assume that solution of the problem (1)-(6) could be found out in the following form: $\mathbf{v}=(v(y, z), 0,0), \mathbf{v}_{g}=\left(v_{g}(y, z), 0,0\right), \rho_{g} \ll \rho$. Accordingly, the gravity does not affect significantly the gas flow, which is induced by a constant longitudinal pressure gradient $\lambda$. The continuity equations in (1) and (2) are satisfied exactly. Accordingly, system (1)-(6) could be rewritten as follows

$$
\begin{aligned}
& \frac{\partial^{2} v}{\partial y^{2}}+\frac{\partial^{2} v}{\partial z^{2}}=\frac{p_{x}-\rho g \sin \beta}{\mu}, \\
& p_{y}=0, \\
& -p_{z}-\rho g \cos \beta=0, \\
& \frac{\partial^{2} v_{g}}{\partial y^{2}}+\frac{\partial^{2} v_{g}}{\partial z^{2}}=-\frac{\lambda}{\mu_{g}}, \\
& p_{g}=p_{0}-\lambda x, p_{0}=\text { const. }
\end{aligned}
$$

Here $p_{0}$ is the pressure in the gas at $x=0$. Conditions (3), (4) and the velocity continuity condition (5) save their form. And projection of the dynamic condition (6) on the $x$ axis gives the equation

$$
\mu\left(-H^{\prime} \frac{\partial v}{\partial y}+\frac{\partial v}{\partial z}\right)=\mu_{g}\left(-H^{\prime} \frac{\partial v_{g}}{\partial y}+\frac{\partial v_{g}}{\partial z}\right),
$$

the projection of the dynamic condition on the normal $\mathbf{n}$ taking into account (11) gives:

$$
p-p_{0}+\lambda x=2 \sigma K+\frac{A}{6 \pi h^{3}} .
$$

Conditions (8) and (9) are satisfied if we assume that in the liquid phase

$$
p=p_{1}-\lambda x+\rho g z \cos \beta, \quad p_{1}=\text { const }
$$

Here $\mathrm{p}_{1}$ - pressure at the liquid - solid interface. Then, from condition (13) and the definition of the mean curvature we obtain equation for the function $z=H(y)$ :

$$
\begin{aligned}
& \frac{H^{\prime \prime}}{\left(1+H^{\prime 2}\right)^{3 / 2}}-\frac{\rho g \cos \beta}{\sigma} H+C_{1}=\frac{A}{6 \pi h^{3} \sigma}, \\
& H(0)=b .
\end{aligned}
$$

Constant $C_{1}=p_{1}-p_{0}$ should be calculated. Hence, the problem is reduced to the determination of the function $z=H(y)$, which specifies the shape of the rivulet, the velocity components in the liquid and gas phases $v(y, z), v_{g}(y, z)$, and the constant $C_{1}$, i.e. the system of Eqs. (7), (10), (15) with conditions (3)-(5), (12), and (16) should be solved numerically. Due to the one-dimensionality of the vectors $\mathbf{v}$ and $\mathbf{v}_{g}$, each of the conditions (3)-(5) is equivalent to the one scalar equality, and the pressure distributions in the liquid and the gas are given by the explicit formulas (11), (14). For the numerical solution of the problem formulated above, it is necessary to specify two constants, namely, the rivulet maximum height $b$ and the longitudinal pressure gradient $\lambda$. If they are known, then after the solution of the problem the liquid and gas flow rates $Q$ and $Q_{g}$ could be found out by the formulas 


$$
Q=\iint_{G_{0}} v d y d z, Q_{g}=\iint_{\Omega_{0}} v_{g} d y d z,
$$

where $G_{0}$ and $\Omega_{0}$ are the cross-sections of areas $G$ and $\Omega$ by the plane $x=$ const. This problem is known as a "direct problem". In the simpler form, this problem was solved in [11]. The velocity field in the gas was not calculated, and the action of the gas flow on the liquid was replaced by the presence of a given constant shear stress directed along the $x$ axis at the interface. But so called "inverse problem" is much more widely used for practical applications, because it allows calculating the values of $b$ and $\lambda$ from a condition of the correspondence of the flow rates calculated using formulas (17) after the solution of the direct problem with the prescribed flow rates. In the present paper, we assume that gas and liquids Reynolds numbers, $\mathbf{R e}=Q \rho / \mu, \mathbf{R e}_{g}=Q_{g} \rho_{g} / \mu_{g}$, are the given parameters and shape of the liquid rivulet should be calculated, so the inverse problem is solved. The numerical solution was conducted as follows. We assume that for some arbitrary $b_{0}$ and $\lambda_{0}$ the solution of the direct problem was obtained, and hence the functions $Q_{0}\left(b_{0}, \lambda_{0}\right)$ and $Q_{0 g}\left(b_{0}, \lambda_{0}\right)$ specifying the values of the flow rates are known. Then the values of unknown $b$ and $\lambda$ could be found from the solution of the system

$$
Q_{0}\left(b_{0}, \lambda_{0}\right)=Q, \quad Q_{0 g}\left(b_{0}, \lambda_{0}\right)=Q_{g} .
$$

Here values Q and $Q_{g}$ are prescribed. Eqs. (19) were solved using the modified Newton's method. On each iteration step, while solving the direct problem, the system (15)-(16) was solved to find the function $z=H(y)$ and then Eqs. (7) and (10), with boundary conditions (3)-(5) and (12) were solved to find the velocity fields $\mathbf{v}, \mathbf{v} g$. The latter problem was solved by a finite-difference method using fractional steps with respect to a fictitious time introduced for the calculations.

\section{Results}

In the numerical calculations, the material constants for the gas phase correspond to the nitrogen, and for the liquid to the FC-72: $\mu=0.735 \times 10^{-3} \mathrm{~kg} / \mathrm{m} \cdot \mathrm{s}, \mu g=17.75 \times 10^{-6} \mathrm{~kg} / \mathrm{m} \cdot \mathrm{s}, \rho=1687.8 \mathrm{~kg} / \mathrm{m}^{3}$, $\rho g=1.2505 \mathrm{~kg} / \mathrm{m}^{3}$, and $\sigma=12.57 \times 10^{-3} \mathrm{~N} / \mathrm{m}, \beta=0, \mathrm{~d}=1,4 \mathrm{~mm}, l=4 \mathrm{~cm}, \mathrm{~T}=20^{\circ} \mathrm{C}, \mathrm{A}=10^{-20} \mathrm{~m}^{2} \mathrm{~kg} / \mathrm{s}^{2}$.

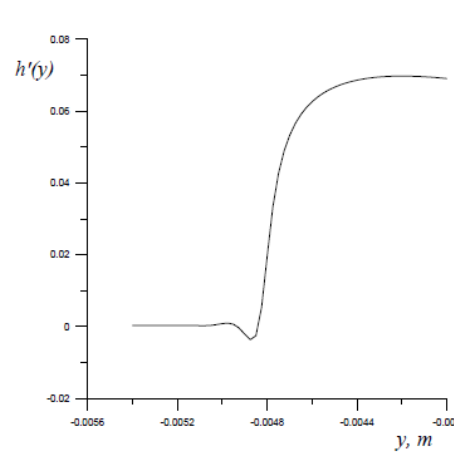

(3)

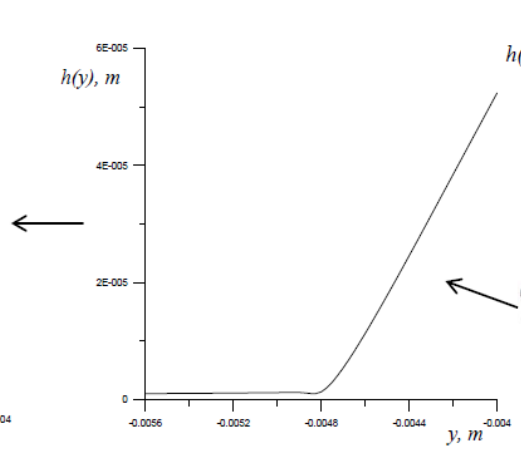

(2)

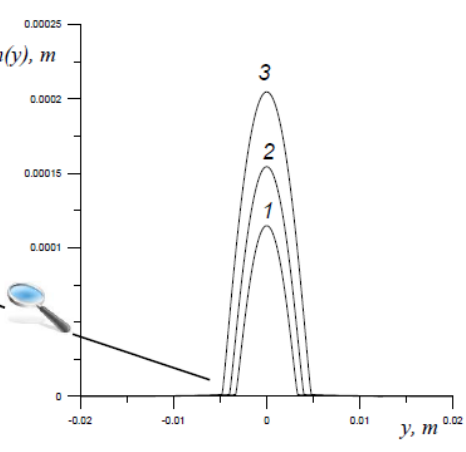

(1)

Figure 2. (1) - Rivulet profiles in cross sections perpendicular to the flow. $\operatorname{Re}=8.1-\operatorname{Re}_{\mathrm{g}}=400 ; 2-\operatorname{Re}_{\mathrm{g}}=200 ; 3$ - $\operatorname{Re}_{g}=100$. (2) - Thin liquid film near the contact line and contact angle. $R e=8, R_{g}=100$. (3) - Derivative of the function $\mathrm{h}(\mathrm{y})$ with respect to $\mathrm{y}$, near the contact line. $\mathrm{Re}=8, \mathrm{Re}_{\mathrm{g}}=100$.

Figure 2, (1) shows the rivulet profiles in cross sections perpendicular to the flow at different gas Reynolds numbers. One can see that decreasing of gas velocity leads to the important change in the rivulet profile. Formally it is assumed that rivulet and thin liquid film fully covers the bottom wall of the channel, but at the rivulet profile clearly visible three-phase contact line and region with 
practically constant inclination could be seen near the thin liquid film areas, Fig.2, (2), and the angle of this inclination could be considered as a contact angle. Region with practically constant derivative of the function $\mathrm{h}(\mathrm{y})$ with respect to $\mathrm{y}$, near the contact line confirms this statement, Fig. 2, (3). Figure 3 shows velocity field in cross section of the channel. The rivulet flow region is clearly visible because the velocity in the liquid is smaller than in the gas. One can see that rivulet formation gives a significant disturbing effect on the gas velocity distribution.

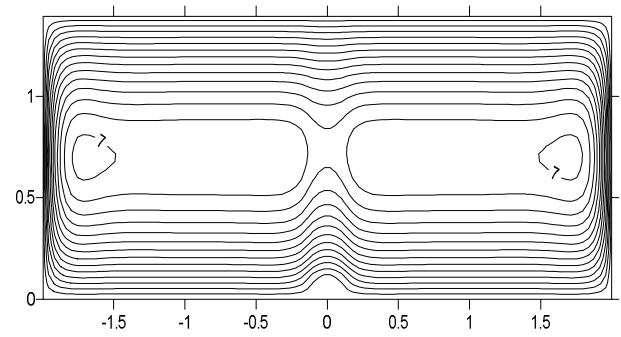

Figure 3. Velocity field in cross section $(\mathrm{m} / \mathrm{s}) . \mathrm{Re}=2, \mathrm{Re}_{\mathrm{g}}=20$.

\section{Conclusion}

For an incompressible and nonisothermal liquid rivulet moved by a gas flow in an inclined minichannel the lubrication-type mathematical model based on the predominant velocity direction approach was built. Action of the van der Waals and capillary forces, as well as gravity and tangential stress at the gas-liquid interface are taking into account. Numerically it is found out that rivulet formation gives a significant disturbing effect on the gas velocity distribution. It is shown that at the rivulet profile clearly visible three-phase contact line and region with practically constant inclination could be seen near the thin liquid film areas, and the angle of this inclination could be considered as a contact angle. Region with practically constant derivative of the function $\mathrm{h}(\mathrm{y})$ with respect to $\mathrm{y}$, near the contact line confirms this statement.

\section{Acknowledgements}

The study was financially supported by the Russian Science Foundation, project no. 14_19_01755. The numerical algorithm was elaborated under the support of RFBR (No 14-08-00163).

\section{References}

1. P. Stephan, C. Brandt, Proc. I Int. Conf. on Microchannels and Minichannels (ed. S.G. Kandlikar), Rochester, NY, 24-25 April (2003)

2. A.Ye. Rednikov, P. Colinet, Langmuir 27, 5 (2011)

3. V. S. Ajaev, E. Ya. Gatapova, O. A. Kabov, Advances in Colloid and Interface Science 228, (2016)

4. A.L. Karchevsky, I.V. Marchuk and O.A. Kabov, Applied Mathematical Modeling 40, (2016)

5. D.V. Zaitsev, A.M. Lozano, H. Auracher, O.A. Kabov, Microgravity science and technology, 19, $71(2007)$

6. V.V.Cheverda, A.Glushchuk, P.Queeckers, S.B.Chikov, O.A.Kabov, Microgravity Science and Technology 25 (2013)

7. G.D. Towell and L.B. Rothfeld, AIChE Journal, 12, 5 (1966) 
8. S.V. Alekseenko, P.I. Geshev, and P.A. Kuibin, Doklady Physics, 42, 5 (1997)

9. M.V. Bartashevich, O.A. Kabov, V.V. Kuznetsov, Fluid Dynamics, 45, 6 (2010)

10. B.V. Deriagin, N.V. Churaev, V.M. Muller (1985, M.: Nauka)

11. T.G. Myers, H.X. Liang, and B. Wetton, International Journal of Non-linear Mechanics 39 (2004) 